\title{
Thread-Modular Verification for Shared-Memory Programs
}

\author{
Cormac Flanagan, Stephen N. Freund, and Shaz Qadeer \\ Compaq Systems Research Center, 130 Lytton Ave., Palo Alto, CA 94301
}

\begin{abstract}
Ensuring the reliability of multithreaded software systems is difficult due to the interaction between threads. This paper describes the design and implementation of a static checker for such systems. To avoid considering all possible thread interleavings, the checker uses assumeguarantee reasoning, and relies on the programmer to specify an environment assumption that constrains the interaction between threads. Using this environment assumption, the checker reduces the verification of the original multithreaded program to the verification of several sequential programs, one for each thread. These sequential programs are subsequently analyzed using extended static checking techniques (based on verification conditions and automatic theorem proving). Experience indicates that the checker is capable of handling a range of synchronization disciplines. In addition, the required environment assumptions are simple and intuitive for common synchronization idioms.
\end{abstract}

\section{Introduction}

Ensuring the reliability of critical software systems is an important but extremely difficult task. A number of useful tools and techniques have been developed for reasoning about sequential systems. Unfortunately, these sequential analysis tools are not applicable to many critical software systems because such systems are often multithreaded. The presence of multiple threads significantly complicates the analysis because of the potential for interference between threads; each atomic step of a thread can influence the subsequent behavior of other threads.

For multithreaded programs, more complex analysis techniques are necessary. The classical assertional approach Ash750G76 Lam77Lam88 requires control predicates at each program point to specify the reachable program states, but the annotation burden for using this approach is high. Some promising tools $\mathrm{DHJ}^{+} 01$ Yah01] use model checking and abstract interpretation to infer the reachable state set automatically, but the need to consider all possible thread interleavings may hinder scaling to large programs.

A more modular and scalable approach is assume-guarantee reasoning, in which each component is verified separately using a specification of the other components MC81.Jon83a]. Several researchers have presented assume-guarantee proof rules (see Section [2), and some verification tools that support assumeguarantee reasoning on hardware have recently appeared [McM97 $\mathrm{AHM}^{+98}$. 
However, tools for assume-guarantee reasoning on realistic software systems do not exist.

In this paper, we describe the design and implementation of a static checker for multithreaded programs, based on an assume-guarantee decomposition. This checker is targeted to the verification of actual implementations of software systems, as opposed to logical models or abstractions of these systems. The checker relies on the programmer to specify, for each thread, an environment assumption that models the interference caused by other threads. This environment assumption is an action, or two-store relation, that constrains the updates to the shared store by interleaved atomic steps of other threads. The atomic steps of each thread are also required to satisfy a corresponding guarantee condition that implies the assumption of every other thread.

Using these assumptions and guarantees, our checker translates each thread into a sequential program that models the behavior of that thread precisely and uses the environment assumption to model the behavior of other threads. Thus, our assume-guarantee decomposition reduces the verification of a program with $n$ threads to the verification of $n$ sequential programs. This thread-modular decomposition allows our tool to leverage extended static checking techniques DLNS98] (based on verification conditions and automatic theorem proving) to check the resulting sequential programs.

We have implemented our checker for multithreaded programs written in the Java programming language [AG96], and we have successfully applied this checker to a number of programs. These programs use a variety of synchronization mechanisms, ranging from simple mutual exclusion locks to more complex idioms found in systems code, including a subtle synchronization idiom used in the distributed file system Frangipani [TML97].

Experience with this implementation indicates that our analysis has the following useful features:

1. It naturally scales to programs with many threads since each thread is analyzed separately.

2. For programs using common synchronization idioms, such as mutexes or reader-writer locks, the necessary annotations are simple and intuitive.

3. Control predicates can be expressed in our analysis by explicating the program counter of each thread as an auxiliary variable. Therefore, theoretically our method is as expressive as the Owicki-Gries method. However, for many common cases, such as those appearing in Section 6, our method requires significantly fewer annotations.

The remainder of the paper proceeds as follows. The following section describes related work on assume-guarantee reasoning and other tools for detecting synchronization errors. Section 3 introduces Plato, an idealized language for parallel programs that we use as the basis for our development. Section 4 provides a formal definition of thread-modular verification. Section [5applies threadmodular reasoning to the problem of invariant verification. Section 6 describes our implementation and its application to a number of example programs. We conclude in Section $[7$. 


\section{Background}

One of the earliest assume-guarantee proof rules was developed by Misra and Chandy MC81 for message-passing systems, and later refined by others (see, for example, Jon89 MM93]). However, their message-passing formulation is not directly applicable to shared-memory software.

Jones [Jon83a Jon83b] gave a proof rule for multithreaded shared-memory programs and used it to manually refine an assume-guarantee specification down to a program. We extend his work to allow the proof obligations for each thread to be checked mechanically by an automatic theorem prover. Stark Sta85] also presented a rule for shared-memory programs to deduce that a conjunction of assume-guarantee specifications hold on a system provided each specification holds individually, but his work did not allow the decomposition of the implementation.

Abadi and Lamport AL95 view the composition of components as a conjunction of temporal logic formulas Lam94] describing them, and they present a rule to decompose such systems. Since threads modifying shared variables cannot be viewed as components in their framework, their work is not directly applicable to our problem. Collette and Knapp [CK95] extended the rule of Abadi and Lamport to the more operational setting of Unity [CM88] specifications.

Alur and Henzinger [AH96] and McMillan McM97] present assume-guarantee proof rules for hardware components. A number of other compositional proof rules not based on assume-guarantee reasoning have also been proposed, such as BKP84CM88 MP95].

Yahav Yah01 describes a method to model check multithreaded programs using a 3-valued logic [SRW99LAS00] to abstract the store. This technique can verify interesting properties of small programs. Păsăreanu et al. PDH99 also describe a model checking tool for compositional checking of finite-state message passing systems. Abraham-Mumm and deBoer AMdB00 sketch a logic for verifying multi-threaded Java programs indirectly via a translation to communicating sequential programs.

A number of tools have been developed for identifying specific synchronization errors in multithreaded programs. These approaches are less general than thread-modular verification and use specific analysis techniques to locate specific errors, such as data races and deadlocks. For example, RCC/Java [FF00] is an annotation-based checker for Java that uses a type system to identify data races [FA99. While this tool is successful at finding errors in large programs, the inability to specify subtle synchronization patterns results in many false alarms [FF01. ESC/Java [LSS99], Warlock [Ste93], and the dynamic testing tool Eraser $\left[\mathrm{SBN}^{+} 97\right]$ are other tools in this category, and are discussed in an earlier paper [FF00.

\section{The Parallel Language Plato}

We present thread-modular verification in terms of the idealized language Plato (parallel language of atomic operations). A Plato program $P$ is a parallel com- 
position $S_{1}|\cdots| S_{n}$ of several statements, or threads. The program executes by interleaving atomic steps of its various threads. The threads interact through a shared store $\sigma$, which maps program variables to values. The sets of variables and values are left intentionally unspecified, as they are mostly orthogonal to our development.

Statements in the Plato language include the empty statement skip, sequential composition $S_{1} ; S_{2}$, the nondeterministic choice construct $S_{1} \square S_{2}$, which executes either $S_{1}$ or $S_{2}$, and the iteration statement $S^{*}$, which executes $S$ some arbitrary number of times.

\section{Plato syntax}

\begin{tabular}{|c|c|c|}
\hline$S \in S t m t::=$ & 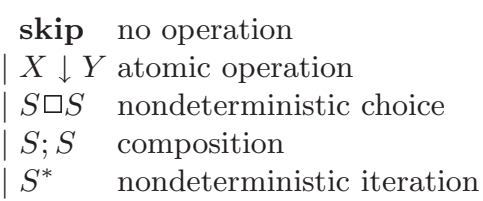 & $\begin{aligned} & P \in \text { Program }::=S_{1}|\cdots| S_{n} \\
& \sigma \in \text { Store }=\text { Var } \rightarrow \text { Value } \\
& X, Y \in \text { Action } \subseteq \text { Store } \times \text { Store }\end{aligned}$ \\
\hline
\end{tabular}

Perhaps the most notable aspect of Plato is that it does not contain constructs for conventional primitive operations such as assignment and lock acquire and release operations. Instead, such primitive operations are combined into a general mechanism called an atomic operation $X \downarrow Y$, where $X$ and $Y$ are actions, or two-store predicates. The action $X$ is a constraint on the transition from the pre-store $\sigma$ to the post-store $\sigma^{\prime}$, and $Y$ is an assertion about this transition.

To execute the atomic operation $X \downarrow Y$, an arbitrary post-store $\sigma^{\prime}$ is chosen that satisfies the constraint $X\left(\sigma, \sigma^{\prime}\right)$. There are two possible outcomes:

1. If the assertion $Y\left(\sigma, \sigma^{\prime}\right)$ holds, then the atomic operation terminates normally, and the execution of the program continues with the new store $\sigma^{\prime}$.

2. If the assertion $Y\left(\sigma, \sigma^{\prime}\right)$ does not hold, then the execution goes wrong.

If no post-store $\sigma^{\prime}$ satisfies the constraint $X\left(\sigma, \sigma^{\prime}\right)$, then the thread is blocked, and the execution can proceed only on the other threads.

In an atomic operation, we write each action as a formula in which primed variables refer to their value in the post-store $\sigma^{\prime}$, and unprimed variables refer to their value in the pre-store $\sigma$. In addition, for any action $X$ and set of variables $V \subseteq \operatorname{Var}$, we use the notation $\langle X\rangle_{V}$ to mean the action that satisfies $X$ and only allows changes to variables in $V$ between the pre-store and the post-store. We abbreviate the common case $\langle X\rangle_{\emptyset}$ to $\langle X\rangle$ and also abbreviate $\langle X\rangle_{\{a\}}$ to $\langle X\rangle_{a}$.

Atomic operations can express many conventional primitives, such as assignment, assert, and assume statements (see below). Atomic operations can also express other primitives, in particular lock acquire and release operations. We assume that each lock is represented by a variable and that each thread has a unique nonzero thread identifier. If a thread holds a lock, then the lock variable contains the corresponding thread identifier; if the lock is not held, then the variable contains zero. Under this representation, acquire and release operations for lock $\mathrm{mx}$ and thread $i$ are shown below. Finally, Plato can also express traditional control constructs, such as if and while statements. 


\section{Expressing conventional constructs in Plato}

\begin{tabular}{|c|c|c|c|c|}
\hline $\begin{aligned} \mathrm{x}:=e & \stackrel{\text { def }}{=} \\
\text { assert } e & \stackrel{\text { def }}{=} \\
\text { assume } e & \stackrel{\text { def }}{=}\end{aligned}$ & $\begin{array}{l}\left\langle\mathrm{x}^{\prime}=e\right\rangle_{\mathrm{x}} \downarrow \text { true } \\
\langle\text { true }\rangle \downarrow e \\
\langle e\rangle \downarrow \text { true }\end{array}$ & $\begin{array}{r}\operatorname{acq}(\mathrm{mx}) \\
\mathrm{rel}(\mathrm{mx}) \\
\text { if }(e)\{S\} \\
\text { while }(e)\{S\}\end{array}$ & $\begin{array}{l}\stackrel{\text { def }}{=} \\
\stackrel{\text { def }}{=} \\
\stackrel{\text { def }}{=} \\
\stackrel{\text { def }}{=}\end{array}$ & $\begin{array}{l}\left\langle\mathrm{mx}=0 \wedge \mathrm{mx}^{\prime}=\mathrm{i}\right\rangle_{\mathrm{mx}} \downarrow \text { true } \\
\left\langle\mathrm{mx}^{\prime}=0\right\rangle_{\mathrm{mx}} \downarrow(\mathrm{mx}=\mathrm{i}) \\
\text { (assume } e ; S) \square(\text { assume } \neg e) \\
(\text { assume } e ; S)^{*} ;(\text { assume } \neg e)\end{array}$ \\
\hline
\end{tabular}

\section{$3.1 \quad$ Formal Semantics}

The execution of a program is defined as an interleaving of the executions of its individual, sequential threads. A sequential state $\Phi$ is either a pair of a store and a statement, or the special state wrong (indicating that the execution went wrong by failing an assertion). The semantics of individual threads is defined via the transition relation $\Phi \rightarrow_{s} \Phi$, defined in the figure below.

A parallel state $\Theta$ is either a pair of a store and a program (representing the threads being executed), or the special state wrong. The transition relation $\Theta \rightarrow p \Theta$ on parallel states executes a single sequential step of an arbitrarily chosen thread. If that sequential step terminates normally, then execution continues with the resulting post-state. If the sequential step goes wrong, then so does the entire execution.

\section{Formal semantics of Plato}

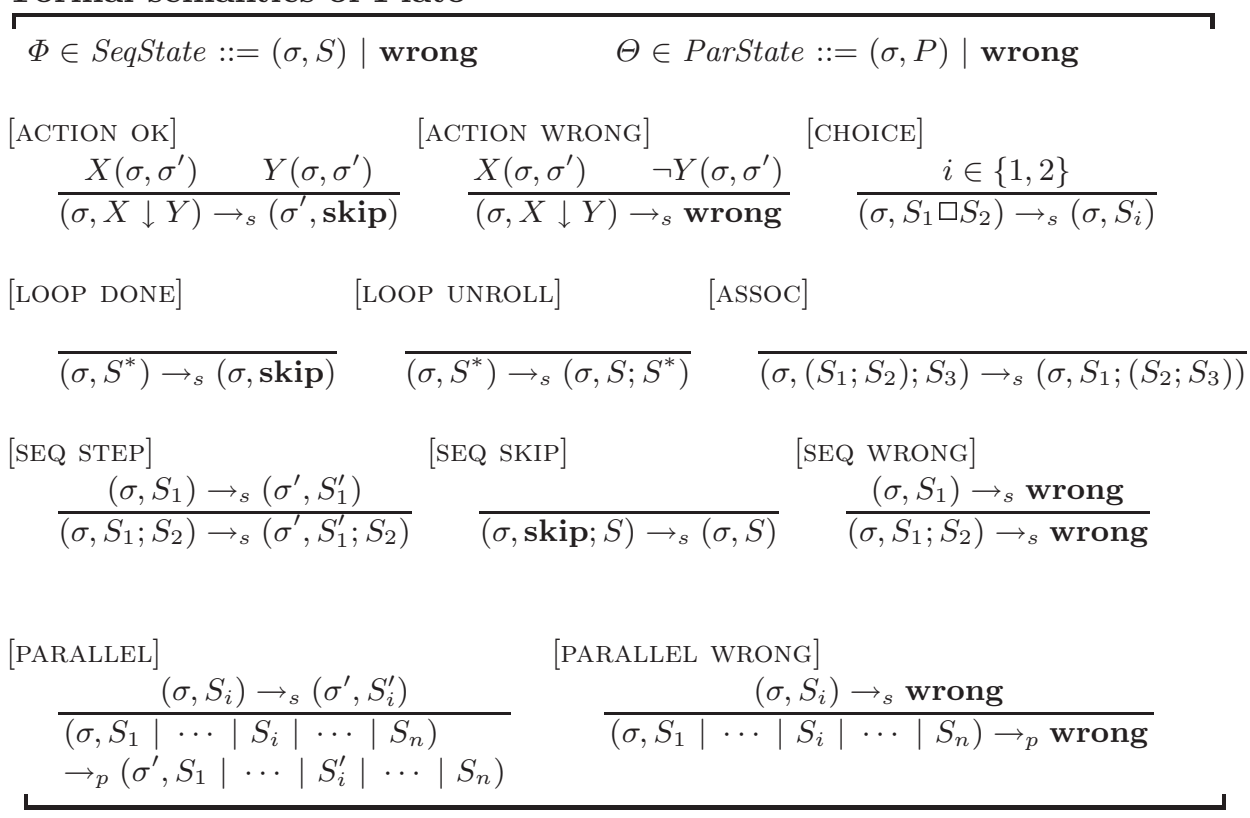




\section{Thread-Modular Verification}

We reason about a parallel program $P=S_{1}|\cdots| S_{n}$ by reasoning about each thread in $P$ separately. For each thread $i$, we specify two actions - an environment assumption $A_{i}$ and a guarantee $G_{i}$. The assumption of a thread is a specification of what transitions may be performed by other threads in the program. The guarantee of a thread is required to hold on every action performed by the thread itself. To ensure the correctness of the assumptions, we require that the guarantee of each thread be stronger than the assumption of every other thread. In addition, to accommodate effect-free transitions, we require each assumption and guarantee to be reflexive. The precise statement of these requirements is as follows:

1. $A_{i}$ and $G_{i}$ are reflexive for all $i \in 1$..n.

2. $G_{i} \subseteq A_{j}$ for all $i, j \in 1$..n such that $i \neq j$.

If these requirements are satisfied, then $\left\langle A_{1}, G_{1}\right\rangle, \ldots,\left\langle A_{n}, G_{n}\right\rangle$ is an assumeguarantee decomposition for $P$.

We next define the translation $\llbracket S \rrbracket_{G}^{A}$ of a statement $S$ with respect to an assumption $A$ and a guarantee $G$. This translation verifies that each atomic operation of $S$ satisfies the guarantee $G$. In addition, the translation inserts the iterated environment assumption $A^{*}$ as appropriate to model atomic steps of other threads.

$$
\begin{aligned}
& \llbracket \bullet \rrbracket: \text { Stmt } \times \text { Action } \times \text { Action } \rightarrow \text { Stmt } \\
& \llbracket \text { skip } \rrbracket_{G}^{A}=A^{*} \\
& \llbracket X \downarrow Y \rrbracket_{G}^{A}=A^{*} ; X \downarrow(Y \wedge G) ; A^{*} \\
& \llbracket S_{1} \square S_{2} \rrbracket_{G}^{A}=A^{*} ;\left(\llbracket S_{1} \rrbracket_{G}^{A} \square \llbracket S_{2} \rrbracket_{G}^{A}\right) \\
& \llbracket S_{1} ; S_{2} \rrbracket_{G}^{A}=\llbracket S_{1} \rrbracket_{G}^{A} ; \llbracket S_{2} \rrbracket_{G}^{A} \\
& \llbracket S^{*} \rrbracket_{G}^{A}=A^{*} ;\left(\left(\llbracket S \rrbracket_{G}^{A} ; A^{*}\right)^{*} ; A^{*}\right)
\end{aligned}
$$

We use this translation and the assume-guarantee decomposition to abstract each thread $i$ of the parallel program $P$ into the sequential program $\llbracket S_{i} \rrbracket_{G_{i}}^{A_{i}}$, called the $i$-abstraction of $P$. For any thread $i$, if $A_{i}$ models the environment of thread $i$ and the sequential $i$-abstraction of $P$ does not go wrong, then we conclude that the corresponding thread $S_{i}$ in $P$ does not go wrong and also satisfies the guarantee $G_{i}$. Thus, if none of the $i$-abstractions go wrong, then none of the threads in $P$ go wrong. This property is formalized by the following theorem; its correctness proof avoids circular reasoning by using induction over time. (An extended report containing the proof of theorems in this paper is in available at http://www.research. compaq. com/SRC/personal/freund/tmv-draft.ps.)

Theorem 1 (Thread-Modular Verification). Let $P=S_{1}|\cdots| S_{n}$ be $a$ parallel program with assume-guarantee decomposition $\left\langle A_{1}, G_{1}\right\rangle, \ldots,\left\langle A_{n}, G_{n}\right\rangle$. For all $\sigma \in$ Store, if $\forall i \in 1 . . n$. $\left(\sigma, \llbracket S_{i} \rrbracket_{G_{i}}^{A_{i}}\right) \nrightarrow_{s_{s}^{*}}$ wrong, then $(\sigma, P) \nrightarrow_{p}^{*}$ wrong.

This theorem allows us to decompose the analysis of a parallel program $S_{1}|\cdots| S_{n}$ into analyses of individual threads by providing an assume-guarantee 
decomposition $\left\langle A_{1}, G_{1}\right\rangle, \ldots,\left\langle A_{n}, G_{n}\right\rangle$. In practice, we only require the programmer to specify reflexive assumptions $A_{1}, \ldots, A_{n}$, and we derive the corresponding reflexive guarantees by

$$
G_{i}=\left(\forall j \in 1 . . n \cdot j \neq i \Rightarrow A_{j}\right)
$$

For all examples we have considered, the natural assumptions are transitive in addition to being reflexive. This allows us to optimize the iterations $A_{i}^{*}$ in each $i$ abstraction to simply the action $A_{i}$. In addition, the $n$ environment assumptions $A_{1}, \ldots, A_{n}$ for a program with $n$ threads can typically be conveniently expressed as a single action parameterized by thread identifier, as shown below.

\subsection{Example}

To illustrate Theorem 1 consider the following program SimpleLock. The program manipulates two shared variables, an integer $\mathrm{x}$ and a lock $\mathrm{mx}$. To synchronize accesses to $\mathrm{x}$, each thread acquires the lock $\mathrm{mx}$ before manipulating $\mathrm{x}$. The correctness condition we would like to verify is that Thread ${ }_{1}$ never goes wrong by failing the assertion $\mathrm{x}>1$.

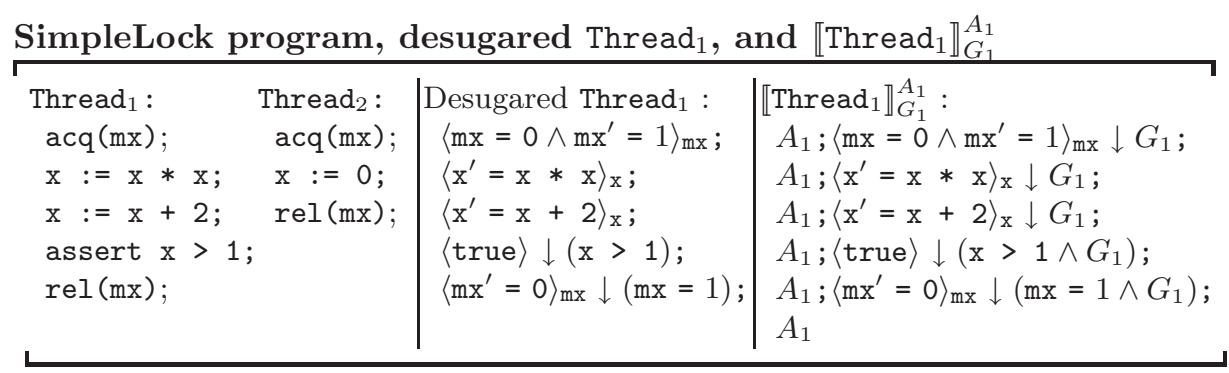

The synchronization discipline in this program is that if a thread holds the lock $\mathrm{mx}$, then the other thread cannot modify either the variable $\mathrm{x}$ or the lock variable $\mathrm{mx}$. This discipline is formalized by the following environment assumption for thread identifier $i \in 1 . .2$ :

$$
A_{i}=\left(\mathrm{mx}=i \Rightarrow \mathrm{mx}^{\prime}=i \wedge \mathrm{x}^{\prime}=\mathrm{x}\right)
$$

The corresponding guarantees are $G_{1}=A_{2}$ and $G_{2}=A_{1}$. Since $A_{1}$ is reflexive and transitive, we can optimize both $A_{1}^{*}$ and $A_{1}^{*} ; A_{1}^{*}$ to $A_{1}$ in the 1-abstraction of SimpleLock, shown above.

Verifying the two $i$-abstractions of SimpleLock is straightforward, using existing analysis techniques for sequential programs. In particular, our checker uses extended static checking to verify that the two sequential $i$-abstractions of SimpleLock do not go wrong. Thus, the hypotheses of Theorem 1 are satisfied, and we conclude that the parallel program SimpleLock does not fail its assertion. 


\section{Invariant Verification}

In the previous section, we showed that the SimpleLock program does not fail its assertion. In many cases, we would also like to show that a program preserves certain data invariants. This section extends thread-modular verification to check data invariants on a parallel program $P=S_{1}|\ldots| S_{n}$. We use Init $\subseteq$ Store to describe the possible initial states of $P$, and we say that a set of states $I$ is an invariant of $P$ with respect to Init if for each $\sigma \in \operatorname{Init}$, if $(\sigma, P) \rightarrow_{p}^{*}\left(\sigma^{\prime}, P^{\prime}\right)$, then $\sigma^{\prime} \in I$.

To show that $I$ is an invariant of $P$, it suffices to show that $I$ holds initially (i.e., Init $\subseteq I$ ), and that $I$ is preserved by each transition of $P$. We prove the latter property using thread-modular verification, where the guarantee $G_{i}$ of each thread satisfies the property

$$
G_{i} \Rightarrow\left(I \Rightarrow I^{\prime}\right)
$$

In this formula, the predicate $I$ denotes the action where $I$ holds in the pre-state, and the post-state is unconstrained; similarly, $I^{\prime}$ denotes the action where the pre-state is unconstrained, and $I$ holds in the post-state. Thus, $I \Rightarrow I^{\prime}$ is the action stating that $I$ is preserved.

The following theorem formalizes the application of thread-modular reasoning to invariant verification.

Theorem 2 (Invariant Verification). Let $P=S_{1}|\cdots| S_{n}$ be a parallel program with assume-guarantee decomposition $\left\langle A_{1}, G_{1}\right\rangle, \ldots,\left\langle A_{n}, G_{n}\right\rangle$, and let Init and $I$ be sets of stores. Suppose:

1. Init $\subseteq I$

2. $\forall i \in 1$..n. $G_{i} \Rightarrow\left(I \Rightarrow I^{\prime}\right)$

3. $\forall i \in 1$..n. $\forall \sigma \in$ Init. $\left(\sigma, \llbracket S_{i} \rrbracket_{G_{i}}^{A_{i}}\right) \nrightarrow_{s}^{*}$ wrong

Then $I$ is an invariant of $P$ with respect to Init.

In practice, we apply this theorem by requiring the programmer to supply the invariant $I$ and the parameterized environment assumption $A_{i}$. We derive the corresponding parameterized guarantee:

$$
G_{i}=\left(\forall j \in 1 . . n . j \neq i \Rightarrow A_{j}\right) \wedge\left(I \Rightarrow I^{\prime}\right)
$$

The guarantee states that each atomic step of a thread satisfies the assumptions of the other threads and also preserves the invariant. Since each step preserves the invariant, we can strengthen the environment assumption to:

$$
B_{i}=A_{i} \wedge\left(I \Rightarrow I^{\prime}\right)
$$

The resulting assume-guarantee decomposition $\left\langle B_{1}, G_{1}\right\rangle, \ldots,\left\langle B_{n}, G_{n}\right\rangle$ is then used in the application of Theorem 2 The first condition of that theorem, that Init $\subseteq I$, can be checked using a theorem prover [Nel81]. The second condition, 
that $\forall i \in 1 . . n . G_{i} \Rightarrow\left(I \Rightarrow I^{\prime}\right)$, follows directly from the definition of $G_{i}$. The final condition (similar to the condition of Theorem 1), that each sequential $i$-abstraction $\llbracket S_{i} \rrbracket_{G_{i}}^{B_{i}}$ does not go wrong from any initial store in Init, can be checked using extended static checking. The following section describes our implementation of an automatic checking tool for parallel programs that supports thread modular and invariant verification.

\section{Implementation and Applications}

We have implemented an automatic checking tool for parallel, shared-memory programs. This checker takes as input a Java program, together with annotations describing appropriate environment assumptions, invariants, and asserted correctness properties. The input program is first translated into an intermediate representation language similar to Plato, and then the techniques of this paper are applied to generate an $i$-abstraction, which is parameterized by the thread identifier $i$.

This $i$-abstraction is then converted into a verification condition Dij75 FS01]. When generating this verification condition, procedure calls are handled by inlining, and loops are translated either using a programmer-supplied loop invariant, or in an unsound but useful manner by unrolling loops some finite number of times [LSS99. The automatic theorem prover Simplify Nel81 is then invoked to check the validity of this verification condition.

If the verification condition is valid, then the parameterized $i$-abstraction does not go wrong, and hence the original Java program preserves the stated invariants and assertions. Alternatively, if the verification condition is invalid, then the theorem prover generates a counterexample, which is then post-processed into an appropriate error message in terms of the original Java program. Typically, the error message either identifies an atomic step that may violate one of the stated invariants or environment assumptions, or identifies an assertion that may go wrong. This assertion may either be explicit, as in the example programs, or may be an implicit assertion, for example, that a dereferenced pointer is never null.

The implementation of our checker leverages extensively off the Extended Static Checker for Java, which is a powerful checking tool for sequential Java programs. For more information regarding ESC/Java, we refer the interested reader to related documents [DLNS98 LSS99 $\mathrm{FLL}^{+} 02$ ].

In the next three subsections, we describe the application of our checker to parallel programs using various kinds of synchronization. Due to space restrictions, these examples are necessarily small, but our checker has also been applied to significantly larger programs. In each of the presented examples, we state the necessary annotations: the assumptions $A_{i}$ for each thread $i$ and the invariant $I$ to be proved. Given these annotations, our tool can automatically verify each of the example programs. For consistency with our earlier development, these programs are presented using Plato syntax. 


\subsection{Dekker's Mutual Exclusion Algorithm}

Our first example is Dekker's algorithm, a classic algorithm for mutual exclusion that uses subtle synchronization.

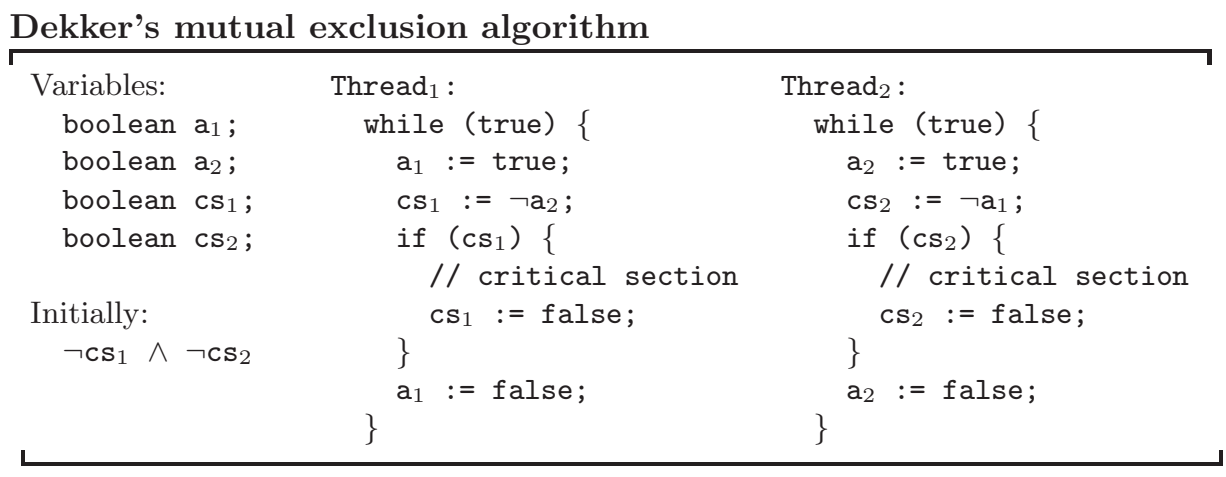

The algorithm uses two boolean variables $\mathrm{a}_{1}$ and $\mathrm{a}_{2}$. We introduce two variables $\mathbf{c s}_{1}$ and $\mathbf{c s}_{2}$, where $\mathbf{c s}_{i}$ is true if thread $i$ is in its critical section. Each Thread ${ }_{i}$ expects that the other thread will not modify $\mathbf{a}_{i}$ and $\mathbf{c s}_{i}$. We formalize this expectation as the assumption:

$$
A_{i}=\left(\mathrm{a}_{i}=\mathrm{a}_{i}^{\prime} \wedge \mathrm{cs}_{i}=\mathrm{cs}_{i}^{\prime}\right)
$$

We would like to verify that the algorithm achieves mutual exclusion, which is expressed as the invariant $\neg\left(\mathbf{c s}_{1} \wedge \mathbf{c s}_{2}\right)$. Unfortunately, this invariant cannot be verified directly. The final step is to strengthen the invariant to

$$
I=\neg\left(\mathrm{cs}_{1} \wedge \mathrm{cs}_{2}\right) \wedge\left(\mathrm{cs}_{1} \Rightarrow \mathrm{a}_{1}\right) \wedge\left(\mathrm{cs}_{2} \Rightarrow \mathrm{a}_{2}\right) .
$$

Using the assumptions $A_{1}$ and $A_{2}$ and the strengthened invariant $I$, our checker verifies that Dekker's algorithm achieves mutual exclusion.

In this example, the environment assumptions are quite simple. The subtlety of the algorithm is reflected in the invariant which had to be strengthened by two conjuncts. In general, the complexity of the assertions needed by our checker reflects the complexity of the synchronization patterns used in program being checked.

\subsection{Reader-Writer Locks}

The next example applies thread-modular reasoning to a reader-writer lock, which can be held in two different modes, read mode and write mode. Read mode is non-exclusive, and multiple threads may hold the lock in that mode. On the other hand, holding the lock in write mode means that no other threads hold the lock in either mode. Acquire operations block when these guarantees cannot be satisfied.

We implement a reader-writer lock using two variables: an integer w, which identifies the thread holding the lock in write mode (or 0 if no such thread 
exists), and an integer set $r$, which contains the identifiers of all threads holding the lock in read mode. The following atomic operations express acquire and release in read and write mode for thread $i$ :

$$
\begin{aligned}
& \operatorname{acq} w \operatorname{write}(\mathrm{w}, \mathrm{r}) \stackrel{\text { def }}{=}\left\langle\mathrm{w}=0 \wedge \mathrm{r}=\emptyset \wedge \mathrm{w}^{\prime}=i\right\rangle_{\mathrm{w}} \\
& \operatorname{acq} \operatorname{read}(\mathrm{w}, \mathrm{r}) \stackrel{\text { def }}{=}\left\langle\mathrm{w}=0 \wedge \mathrm{r}^{\prime}=\mathrm{r} \cup\{i\}\right\rangle_{\mathrm{r}} \\
& \text { rel_write }(\mathrm{w}, \mathrm{r}) \stackrel{\text { def }}{=}\left\langle\mathrm{w}^{\prime}=0\right\rangle_{\mathrm{w}} \downarrow(\mathrm{w}=i) \\
& \operatorname{rel} \operatorname{read}(\mathrm{w}, \mathrm{r}) \stackrel{\text { def }}{=}\left\langle\mathrm{r}^{\prime}=\mathrm{r} \backslash\{i\}\right\rangle_{\mathrm{r}} \downarrow(i \in \mathrm{r})
\end{aligned}
$$

For a thread to acquire the lock in write mode, there must be no writer and no readers. Similarly, to acquire the lock in read mode, there must be no writer, but there may be other readers, and the result of the acquire operation is to put the thread identifier into the set $r$. The release operations are straightforward. All of these lock operations respect the following data invariant $R W I$ and the environment assumption $R W A_{i}$ :

$$
\begin{aligned}
R W I & =(\mathrm{r}=\emptyset \vee \mathrm{w}=0) \\
R W A_{i} & =\left(\mathrm{w}=i \Leftrightarrow \mathrm{w}^{\prime}=i\right) \wedge\left(i \in \mathrm{r} \Leftrightarrow i \in \mathrm{r}^{\prime}\right)
\end{aligned}
$$

We illustrate the analysis of reader-writer locks by verifying the following program, in which the variable $\mathrm{x}$ is guarded by the reader-writer lock. Thread 2 asserts that the value of $\mathrm{x}$ is stable while the lock is held in read mode, even though Thread $_{1}$ mutates $\mathrm{x}$ while the lock is held in write mode.

\section{Reader-writer lock example}

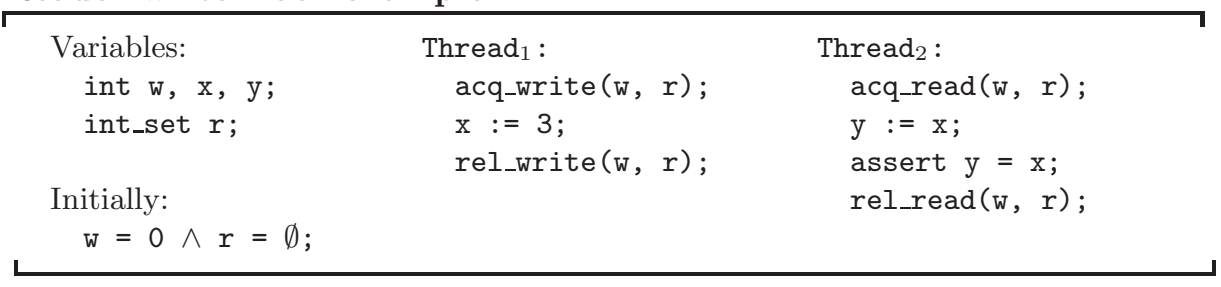

The appropriate environment assumption for this program

$$
A_{i}=R W A_{i} \wedge\left(i \in \mathrm{r} \Rightarrow \mathrm{x}=\mathrm{x}^{\prime}\right) \wedge\left(i=2 \Rightarrow \mathrm{y}=\mathrm{y}^{\prime}\right)
$$

states that (1) each thread $i$ can assume the reader-writer assumption $R W A_{i}$, (2) if thread $i$ holds the lock in read mode, then $\mathrm{x}$ cannot be changed by another thread, and (3) the variable y is modified only by $\operatorname{Thread}_{2}$. This environment assumption, together with the data invariant $R W I$, is sufficient to verify this program using our checker.

Although the reader-writer lock is more complex than the mutual-exclusion lock described earlier, the additional complexity of the reader-writer lock is localized to the annotations $R W A_{i}$ and $R W I$ that specify the lock implementation. Given these annotations, it is encouraging to note that the additional annotations required to verify reader-writer lock clients are still straightforward. 


\subsection{Time-Varying Mutex Synchronization}

We now present a more complex example to show the power of our checker. The example is derived from a synchronization idiom found in the Frangipani file system [TML97.

For each file, Frangipani keeps a data structure called an inode that contains pointers to disk blocks that hold the file data. Each block has a busy bit indicating whether the block has been allocated to an inode. Since the file system is multithreaded, these data structures are guarded by mutexes. In particular, distinct mutexes protect each inode and each busy bit. However, the mutex protecting a disk block depends on the block's allocation status. If a block is unallocated (its busy bit is false), the mutex for its busy bit protects it. If the block is allocated (its busy bit is true), the mutex for the owning inode protects it. The following figure shows a highly simplified version of this situation.

\section{Time-varying mutex program}

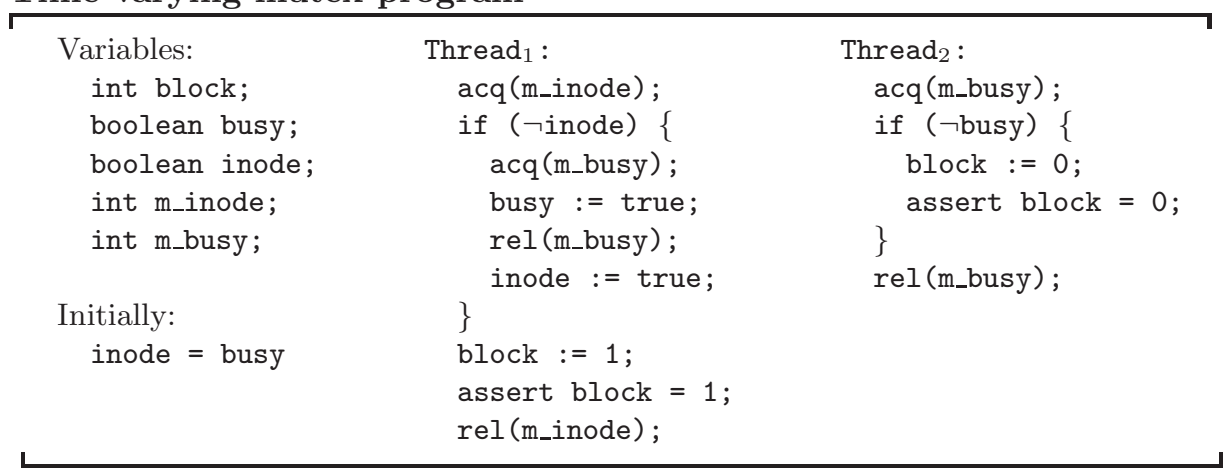

The program contains a single disk block, represented by the integer variable block, and uses a single bit busy to store the block's allocation status. There is a single inode whose contents have been abstracted to a bit indicating whether the inode has allocated the block. The two mutexes m_inode and m_busy protect the variables inode and busy, respectively.

The program contains two threads. Thread ${ }_{1}$ acquires the mutex m_inode, allocates the block if it is not allocated already, and sets block to 1 . Since Thread $_{1}$ is holding the lock on the inode that has allocated the block, the thread has exclusive access to the block contents. Thus, the subsequent assertion that the block value remains 1 should never fail.

Thread $_{2}$ acquires the mutex m_busy. If busy is false, the thread sets block to 0 and asserts that the value of block is 0 . Since Thread 2 holds the lock on busy when the block is unallocated, the thread should have exclusive access to block, and the assertion should never fail.

We now describe annotations necessary to prove that the assertions always hold. First, the lock m_inode protects inode, and the lock m_busy protects busy:

$$
\begin{aligned}
J_{i}= & \left(\text { m_inode }=i \Rightarrow\left(\operatorname{m\_ inode}{ }^{\prime}=i \wedge \text { inode }^{\prime}=\text { inode }\right)\right) \wedge \\
& \left(\text { m_busy }=i \Rightarrow\left(\text { m_busy }^{\prime}=i \wedge \text { busy }^{\prime}=\text { busy }\right)\right)
\end{aligned}
$$


In addition, if busy is true, then block is protected by m_inode; otherwise, block is protected by m_busy:

$$
\begin{aligned}
K_{i}= & \left(\text { busy } \wedge \text { m_inode }=i \Rightarrow \text { block }=\mathrm{block}^{\prime}\right) \wedge \\
& \left(\neg \text { busy } \wedge \text { m_busy }=i \Rightarrow \text { block }=\operatorname{block}^{\prime}\right)
\end{aligned}
$$

Finally, the busy bit must be set when the inode has allocated the block. Moreover, the busy bit can be reset only by the thread that holds the lock on the inode. We formalize these requirements as the invariant $I$ and the assumption $L_{i}$ respectively.

$$
\begin{aligned}
& I=(\text { m_inode }=0 \wedge \text { inode }) \Rightarrow \text { busy } \\
& L_{i}=(\text { m_inode }=i \wedge \text { busy }) \Rightarrow \text { busy }^{\prime}
\end{aligned}
$$

With these definitions, the complete environment assumption for each thread $i$ is:

$$
A_{i}=J_{i} \wedge K_{i} \wedge L_{i}
$$

Given $A_{i}$ and $I$, our checker is able to verify that the assertions in this program never fail.

This example illustrates the expressiveness of our checker. By comparison, previous tools for detecting synchronization errors Ste93 $\mathrm{SBN}^{+} 97$ FF00 have been mostly limited to finding races in programs that only use simple mutexes (and, in some cases, reader-writer locks). However, operating systems and other large-scale systems tend to use a variety of additional synchronization mechanisms, some of which we have described in the last few sections. Other synchronization idioms include binary and counting semaphores, producer-consumer synchronization, fork-join parallelism, and wait-free non-blocking algorithms. Our experience to date indicates that our checker has the potential to handle many of these synchronization disciplines. Of course, the more subtle synchronization disciplines may require more complex annotations, and it may be difficult to check the verification conditions resulting from particularly complex programs or synchronization disciplines.

\section{Conclusions}

The ability to reason about the correctness of large, multithreaded programs is essential to ensure the reliability of such systems. One natural strategy for decomposing such verification problems is procedure-modular verification, which has enjoyed widespread use in a variety of program analysis techniques for many years. Instead of reasoning about a call-site by inlining the corresponding procedure body, procedure-modular verification uses some specification of that procedure, for example, a type signature or a precondition/postcondition pair.

A second, complementary decomposition strategy is assume-guarantee decomposition [Jon83a], which avoids the need to consider all possible interleavings of the various threads explicitly. Instead, each thread is analyzed separately, with an environment assumption providing a specification of the behavior of the other program threads. 
This paper presents an automatic checker for multithreaded programs, based on an assume-guarantee decomposition. The checker relies on the programmer to provide annotations describing the environment assumption of each thread. A potential concern with any annotation-based analysis technique is the overhead of providing such annotations. Our experience applying our checker to a number of example programs indicates that this annotation overhead is moderate. In particular, for many common synchronization idioms, the necessary environment assumptions are simple and intuitive. The environment assumption may also function as useful documentation for multithreaded programs, providing benefits similar to (formal or informal) procedure specifications.

We believe that verification of large, multithreaded programs requires the combination of both thread-modular and procedure-modular reasoning. However, specifying a procedure in a multithreaded program is not straightforward. In particular, because other threads can observe intermediate states of the procedure's computation, a procedure cannot be considered to execute atomically and cannot be specified as a simple precondition/postcondition pair. Combining thread-modular and procedure-modular reasoning appropriately is an important area for future work. Some preliminary steps in this direction are described in a related technical report [FQS02].

Acknowledgements We would like to thank Leslie Lamport, Rustan Leino, and Jim Saxe for valuable feedback on an early version of these ideas; the ESC/Java team who provided the infrastructure on which our checker is based; and Sanjit Seshia for helping with the implementation of the checker.

\section{References}

AG96. K. Arnold and J. Gosling. The Java Programming Language. AddisonWesley, 1996. 263

AH96. R. Alur and T.A. Henzinger. Reactive modules. In Proceedings of the 11th Annual Symposium on Logic in Computer Science, pages 207-218. IEEE Computer Society Press, 1996. 264

$\mathrm{AHM}^{+}$98. R. Alur, T.A. Henzinger, F.Y.C. Mang, S. Qadeer, S.K. Rajamani, and S. Tasiran. Mocha: Modularity in model checking. In A. Hu and M. Vardi, editors, CAV 98: Computer Aided Verification, LNCS 1427, pages 521-525. Springer-Verlag, 1998. 262

AL95. M. Abadi and L. Lamport. Conjoining specifications. ACM Transactions on Programming Languages and Systems, 17(3):507-534, 1995. 264

AMdB00. E. Abraham-Mumm and F. S. de Boer. Proof-outlines for threads in java. In CONCUR 2000: Theories of Concurrency, 2000. [264]

Ash75. E.A. Ashcroft. Proving assertions about parallel programs. Journal of Computer and System Sciences, 10:110-135, January 1975. 262

BKP84. H. Barringer, R. Kuiper, and A. Pnueli. Now you may compose temporallogic specifications. In Proceedings of the 16th Annual Symposium on Theory of Computing, pages 51-63. ACM Press, 1984. 264

CK95. P. Collette and E. Knapp. Logical foundations for compositional verification and development of concurrent programs in Unity. In Algebraic Methodology and Software Technology, LNCS 936, pages 353-367. Springer-Verlag, 1995. 264 
CM88. K.M. Chandy and J. Misra. Parallel Program Design: A Foundation. Addison-Wesley Publishing Company, 1988. 264, 264

$\mathrm{DHJ}^{+}$01. M. Dwyer, J. Hatcliff, R. Joehanes, S. Laubach, C. Pasareanu, Robby, W. Visser, and H. Zheng. Tool-supported program abstraction for finitestate verification. In Proceedings of the 23rd International Conference on Software Engineering, 2001. 262

Dij75. E.W. Dijkstra. Guarded commands, nondeterminacy, and formal derivation of programs. Communications of the ACM, 18(8):453-457, 1975. 270

DLNS98. D. L. Detlefs, K. R. M. Leino, C. G. Nelson, and J. B. Saxe. Extended static checking. Research Report 159, Compaq Systems Research Center, December 1998. 263 270

FA99. C. Flanagan and M. Abadi. Types for safe locking. In Proceedings of European Symposium on Programming, pages 91-108, March 1999. 264]

FF00. C. Flanagan and S.N. Freund. Type-based race detection for Java. In Proceedings of the SIGPLAN Conference on Programming Language Design and Implementation, pages 219-232, 2000. 264, 264, 274]

FF01. C. Flanagan and S.N. Freund. Detecting race conditions in large programs. In Workshop on Program Analysis for Software Tools and Engineering, pages 90-96, June 2001. 264

$\mathrm{FLL}^{+}$02. C. Flanagan, K.R.M. Leino, M. Lillibridge, C.G. Nelson, J.B. Saxe, and R. Stata. Extended static checking for Java. Research Report 178, Compaq Systems Research Center, February 2002. 270

FQS02. C. Flanagan, S. Qadeer, and S. Seshia. A modular checker for multithreaded programs. Technical Note 02-001, Compaq Systems Research Center, 2002. 275

FS01. C. Flanagan and J.B. Saxe. Avoiding exponential explosion: Generating compact verification conditions. In Conference Record of the 28th Annual ACM Symposium on Principles of Programming Languages, pages 193-205. ACM, January 2001. 270

Jon83a. C. B. Jones. Tentative steps toward a development method for interfering programs. ACM Transactions on Programming Languages and Systems, 5(4):596-619, 1983. 262,264, 274

Jon83b. C.B. Jones. Specification and design of (parallel) programs. In R. Mason, editor, Information Processing, pages 321-332. Elsevier Science Publishers B. V. (North-Holland), 1983. 264

Jon89. B. Jonsson. On decomposing and refining specifications of distributed systems. In J.W. de Bakker, W.-P. de Roever, and G. Rozenberg, editors, Stepwise Refinement of Distributed Systems: Models, Formalisms, Correctness, Lecture Notes in Computer Science 430, pages 361-385. Springer-Verlag, 1989. 264

Lam77. L. Lamport. Proving the correctness of multiprocess programs. IEEE Transactions on Software Engineering, SE-3(2):125-143, 1977. 262

Lam88. L. Lamport. Control predicates are better than dummy variables. ACM Transactions on Programming Languages and Systems, 10(2):267-281, April 1988. 262

Lam94. L. Lamport. The Temporal Logic of Actions. ACM Transactions on Programming Languages and Systems, 16(3):872-923, 1994. 264

LAS00. T. Lev-Ami and M. Sagiv. TVLA: A system for implementing static analyses. In Proceedings of the Static Analysis Symposium, pages 280-301, 2000. 264 
LSS99. K. R. M. Leino, J. B. Saxe, and R. Stata. Checking Java programs via guarded commands. In Bart Jacobs, Gary T. Leavens, Peter Müller, and Arnd Poetzsch-Heffter, editors, Formal Techniques for Java Programs, Technical Report 251. Fernuniversität Hagen, May 1999. 264, 270, 270

MC81. J. Misra and K.M. Chandy. Proofs of networks of processes. IEEE Transactions on Software Engineering, SE-7(4):417-426, 1981. 262, 264]

McM97. K.L. McMillan. A compositional rule for hardware design refinement. In O. Grumberg, editor, CAV 97: Computer Aided Verification, Lecture Notes in Computer Science 1254, pages 24-35. Springer-Verlag, 1997. 262, 264

MM93. A. Mokkedem and D. Mery. On using a composition principle to design parallel programs. In Algebraic Methodology and Software Technology, pages 315-324, 1993. 264

MP95. Z. Manna and A. Pnueli. Temporal Verification of Reactive Systems: Safety. Springer-Verlag, 1995. 264

Nel81. C. G. Nelson. Techniques for program verification. Technical Report CSL81-10, Xerox Palo Alto Research Center, 1981. [269] [270]

OG76. S. Owicki and D. Gries. An axiomatic proof technique for parallel programs. Acta Informatica, 6(4):319-340, 1976. [262

PDH99. C.S. Păsăreanu, M.B. Dwyer, and M. Huth. Assume-guarantee model checking of software: A comparative case study. In Theoretical and Practical Aspects of SPIN Model Checking, Lecture Notes in Computer Science 1680, 1999. 264

$\mathrm{SBN}^{+}$97. S. Savage, M. Burrows, C.G. Nelson, P. Sobalvarro, and T.A. Anderson. Eraser: A dynamic data race detector for multithreaded programs. ACM Transactions on Computer Systems, 15(4):391-411, 1997. 264 274

SRW99. M. Sagiv, T. Reps, and R. Wilhelm. Parametric shape analysis via 3valued logic. In Conference Record of the Twenty-Sixth ACM Symposium on Principles of Programming Languages, pages 105-118, 1999. 264

Sta85. E.W. Stark. A proof technique for rely/guarantee properties. In Proceedings of the 5th Conference on Foundations of Software Technology and Theoretical Computer Science, Lecture Notes in Computer Science 206, pages 369-391. Springer-Verlag, 1985. 264

Ste93. N. Sterling. WARLOCK - a static data race analysis tool. In USENIX Technical Conference Proceedings, pages 97-106, Winter 1993. 264, 274,

TML97. C.A. Thekkath, T. Mann, and E.K. Lee. Frangipani: A scalable distributed file system. In Proceedings of the 16th ACM Symposium on Operating Systems Principles, pages 224-237, October 1997. 263, 273]

Yah01. E. Yahav. Verifying safety properties of concurrent Java programs using 3-valued logic. In Proceedings of the 28th Symposium on Principles of Programming Languages, pages 27-40, January 2001. [262, 264] 